\title{
Using Recorded Values for Bounding the Minimum Completion Time in Multiprocessors
}

\author{
Lars Lundberg and Håkan Lennerstad
}

\begin{abstract}
The way the processes in a parallel program are scheduled on the processors of a multiprocessor system affects the performance significantly. Finding a schedule of processes to processors which results in minimum completion time is NP-hard. Therefore, one has to resort to heuristic schedules. However, it is often difficult to determine if a specific schedule is close to the optimal case or if it is worthwhile to look for other schedules.

Based on information from previous executions of the parallel program, we present a formula for an upper bound on the minimum completion time of the program. The bound is a function of a set of parameters. Some of these parameters are obtained from the previous executions of the program and the others describe the target multiprocessor architecture for which we want to bound the minimum completion time. The bound is optimal when it is based on information from one previous execution. Using these results, we are able to decide if a certain schedule is close to optimal or if it is worthwhile to look for other schedules. This is demonstrated by evaluating the completion time of a specific schedule of a particular program. The proofs used for obtaining the bound are based on program transformations and combinatorial mathematics.
\end{abstract}

Index Terms-Parallel program scheduling, optimal performance bounds, multiprocessors with clusters, synchronizing processes, information from previous executions.

\section{INTRODUCTION}

$\mathrm{M}$ ULTIPROCESSORS are now becoming commercially available, and most modern programming environments support parallel programming, e.g., the tasking concept in Ada, the thread concept in Java, and the Sun Solaris operating system. The way the processes in the parallel program are scheduled to the processors of the multiprocessor system affects the performance significantly. Some multiprocessor systems allow processes to be relocated from one processor to another at run-time. In other systems, run-time relocation of processes is prohibited or only allowed within certain groups of processors, e.g., within processor clusters.

We look at systems where a parallel program, consisting of a number of processes, is executed on a multiprocessor. The processors in the system are grouped into equally sized clusters (see Fig. 1). A process can only be executed by the processors in the cluster where it was created. The performance criterion is the completion time of the program.

Using nonoptimal schedules of processes to processors may result in very poor performance. Consider a program consisting of three independent chains containing three processes each (see Fig. 2). The processes are created in such an order that the first chain contains the first three processes, the second chain contains processes four to six, and the last chain contains processes seven to nine. The first process in each chain accepts data from an external source.

- L. Lundberg is with the Department of Computer Science, University of Karlskrona/Ronneby, S-372 25 Ronneby, Sweden.

E-Mail: Lars.Lundberg@ide.hk-r.se.

- H. Lennerstad is with the Deparment of Mathematics, University of Karlskrona/Ronneby, S-371 79 Karlskrona, Sweden.

E-mail: Hakan.Lennerstad@itm.hk-r.se.

Manuscript received 11 Nov. 1996.

For information on obtaining reprints of this article, please send e-mail to: tpds@computer.org, and reference IEEECS Log Number 100337.
The middle process performs a number of transformations on the data, and the last process sends the transformed data on to some other system. The processing in each chain is totally dominated by the middle process, which accounts for 90 percent of the total execution time in the chain. The first and the last process account for 5 percent each. All chains perform the same amount of work.

If we schedule this program on a system with three clusters containing one processors each, we could consider using a naive approach, where process number $x$ is scheduled to cluster $(x \bmod 3)+1$, i.e., processes 1,4 , and 7 are scheduled to cluster one, processes 2,5 , and 8 , are scheduled to cluster two, and processes 3, 6, and 9 to cluster three. Using this schedule, 90 percent of the total amount of work is scheduled to cluster two, whereas clusters one and three only get 5 percent each. If each chain had been scheduled to its own cluster, the work would have been evenly distributed, and the performance of the system would have been almost three times better. Consequently, it is important to find good schedules of processes to processors.

Finding a schedule of processes to processors which results in minimum completion time, i.e., maximum performance, is NP-hard [6]. Therefore, one has to resort to heuristic schedules. Unless a tight upper bound on the minimum completion time is known, it is difficult to determine if a specific schedule is close to the optimal case or if it is worthwhile to look for other schedules. This paper shows that based on a set of recorded values, it is possible to calculate such a bound.

The rest of this paper is structured in the following way. In Section 2, we introduce the definitions necessary for reading this paper. Specifically, the bounds $(t(n, q, \bar{\theta}, \bar{\sigma}, \bar{y}, k, u, \psi)$ and $s(q, \bar{\theta}, \bar{\sigma}, \bar{y}, k, u, \psi))$, which are the main results are 


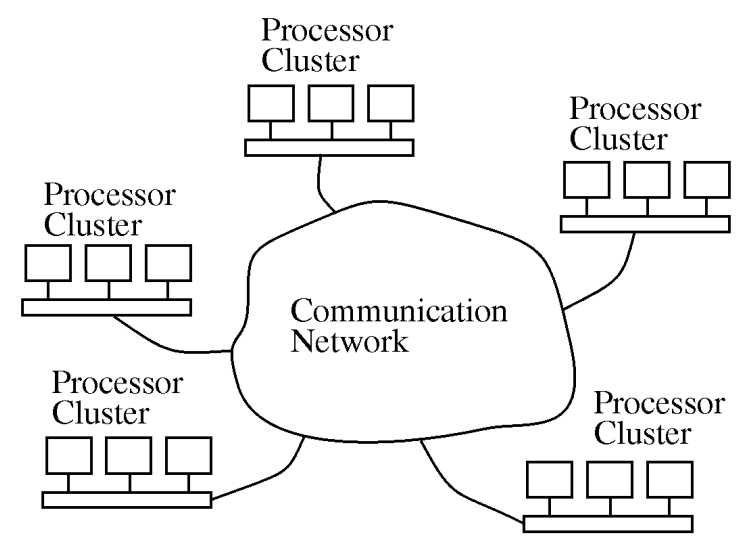

Fig. 1. A multiprocessor with clusters.

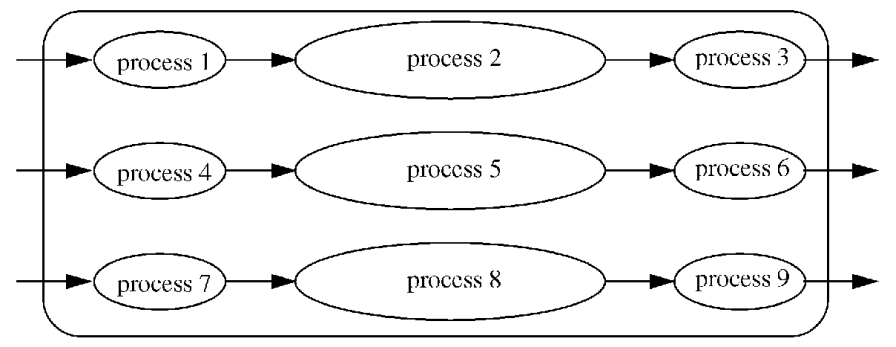

Fig. 2. A parallel program with nine processes.

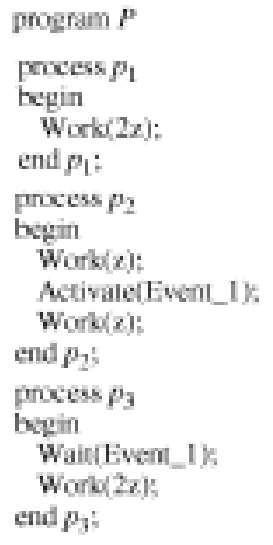

Fig. 3. Textual and graphical representation of a parallel program $P$.

defined in Section 2. In Section 3, the applicability of the results is demonstrated by bounding the performance of a heuristic scheduling algorithm. Section 4 gives a short overview of the methods used for obtaining $(t(n, q, \bar{\theta}, \bar{\sigma}, \bar{y}, k, u, \psi)$ and $s(q, \bar{\theta}, \bar{\sigma}, \bar{y}, k, u, \psi))$. Sections 5,6 , and 7 contain the proofs and transformations necessary for obtaining the bounds. Section 8 compares our results with previous performance bounds and discusses the applicability of the work. Section 9 concludes the paper.

\section{Problem Definition and Results}

A parallel program consists of a set of sequential processes. A process can be either Blocked, Ready, or Running. Processes in the Ready and Running states are referred to as active processes. The execution of a process is controlled by two synchronization primitives: Wait(Event) and Activate(Event), where Event couples a certain Activate to a certain Wait. When a process executes an Activate on an event, we say that the event has occurred. If a process executes a Wait on an event which has not yet occurred, that process becomes blocked until another process executes an Activate on the same event. However, a process executing a Wait on an event which already has occurred does not become blocked. Each process can be represented as a list of sequential segments, which are separated by a Wait or an Activate (see Fig. 3). We assume that, for each process, the length and order of the sequential segments are independent of the way processes are scheduled. All processes are created at the start of the execution. Some processes may, however, be initially blocked by a Wait. 

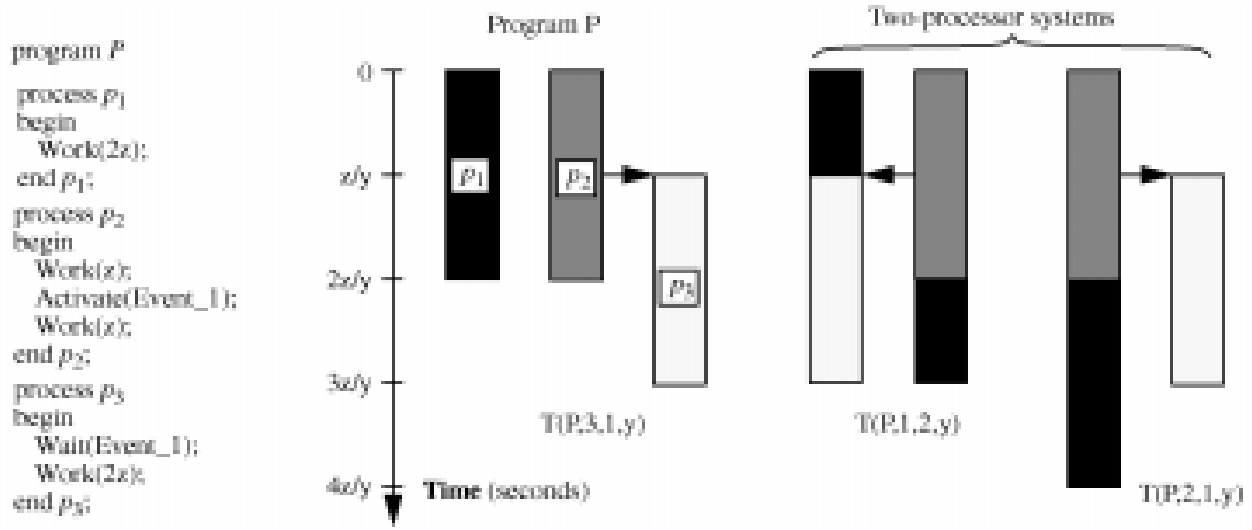

Fig. 4. The minimal completion times for two different systems for a parallel program consisting of three processes.

In multiprocessors with clusters, processors of equal speed are connected in a two-level hierarchy. At the lowest level, processors are connected in equally sized clusters. These clusters are connected via a communication network. Processes may not be relocated from one cluster to another. They may, however, be relocated between the processors in a cluster. We assume that only one program may execute on the system at the same time. Moreover, we disregard overhead for process synchronization, context switching, and relocation (these assumptions are discussed in Sections 8 and 9). Under these conditions, the minimal completion time for a program $P$, using a system with $k$ clusters, each containing $u$ processors with processor speed $y$, is denoted $T(P, k, u, y)$.

The left part of Fig. 4 shows a parallel program with three processes $\left(p_{1}, p_{2}\right.$, and $\left.p_{3}\right)$. Work $(z)$ denotes processing of $z$ instructions. Process $p_{3}$ cannot start its execution before $p_{2}$ has executed for $z / y$ seconds, using a processor with a speed of $y$ instructions per second. This dependency is represented with a Wait on Event_1 in $p_{3}$ and an Activate on the same event in $p_{2}$. The total amount of work performed by the program is denoted $Z$. In the program shown in Fig. 4, $Z=6 z$, i.e., each of the three processes execute $2 z$ instructions. Obviously, $T\left(P, k, u, y_{1}\right) / y_{2}=T\left(P, k, u, y_{2}\right) / y_{1}$, e.g., if $T\left(P, k, u, y_{1}\right)=4 z / y_{1}$, then $T\left(P, k, u, y_{2}\right)=4 z / y_{2}$ and, consequently, $T\left(P, k, u, y_{1}\right) / y_{2}=T\left(P, k, u, y_{2}\right) / y_{1}=4 z /\left(y_{1} * y_{2}\right)$.

The right part of the figure shows a graphical representation of $P$ and two schedules resulting in minimum completion time for a system with one cluster containing two processors and for a system with two clusters containing one processor each. There are two processors in both systems, but in the rightmost case processes may not be relocated from one processor to another. As indicated in Fig. 4, the minimum completion time never decreases when process relocation is prohibited. The completion time of a program $P$ using schedule $A$ and a multiprocessor with $k$ clusters containing $u$ processors each, with processor speed $y$, is denoted $T(P, k, u, y, A)$. Consequently, $T(P, k, u, y)=\min _{A}$ $T(P, k, u, y, A)$.

When there is only one cluster, it is relatively easy to find a schedule for which the completion time is close to the optimal case. In fact, the completion time using a schedule in which no processor is idle when there are processes in the Ready state, e.g., self-scheduling using one common ready queue for all processors, is always within a factor of two from the optimal case [4]. In most cases, the difference between the completion time using self-scheduling and the minimum completion time is marginal.

When there is more than one cluster, the problem of finding a schedule with near minimum completion time becomes much more difficult. Having executed a parallel program on a system containing more than one cluster, it is very hard to determine if the obtained completion time is close to the minimum completion time or if it is worthwhile to look for other schedules. However, if we are able to find an upper bound on the minimum completion time and if the completion time using schedule $A$ is above this bound, we know that there are other schedules with shorter completion time, and it is consequently worthwhile to look for them.

Assume that we have executed the program on a set of $q$ multiprocessor systems with $\theta_{1}, \ldots, \theta_{q}$ processors, respectively, $(\theta i \leq \theta i+1)$ using some, possibly unknown, schedules $A_{1}, \ldots, A_{q}$. The speed of each processor in multiprocessor $i$ is denoted $y_{i}$. These executions result in a vector of completion time values $\bar{\sigma}$, i.e., $\sigma_{i}=T\left(P, 1, \theta_{i}, y_{i}, A_{i}\right)(1 \leq i \leq q)$. Consequently, the previous executions of the program is defined by the five parameters $n, q, \bar{\theta}, \bar{\sigma}$, and $\bar{y}$ (see Fig. 5), where $\bar{\theta}=\theta_{1}, \ldots, \theta_{q}, \bar{\sigma}=\sigma_{1}, \ldots, \sigma_{q}$, and $\bar{y}=y_{1}, \ldots, y_{q}$.

We define the function $t(n, q, \bar{\theta}, \bar{\sigma}, \bar{y}, k, u, \psi)$ such that, for any program $P$ with $n$ processes and a completion time vector $\bar{\sigma}$ of length $q$ corresponding to the vectors $\bar{\theta}$ and $\bar{y}$ :

$$
T(P, k, u, \psi) \leq t(n, q, \bar{\theta}, \bar{\sigma}, \bar{y}, k, u, \psi),
$$

i.e., $t(n, q, \bar{\theta}, \bar{\sigma}, \bar{y}, k, u, \psi)$ is an upper bound on the minimum completion time of $P$ (represented by $n, q, \bar{\theta}, \bar{\sigma}$, and $\bar{y}$, see Fig. 5) using a multiprocessor with $k$ clusters each containing $u$ processors of speed $\psi$. If the completion time using some schedule is above this value, we know that there are other schedules which result in a shorter execution time, i.e., it is worthwhile to look for other schedules.

When $q=1, t(n, q, \bar{\theta}, \bar{\sigma}, \bar{y}, k, u, \psi)$ is optimal, i.e. , there is a program $P$ with $n$ processes and a schedule $A_{1}$, such that $\sigma_{1}=$ $T\left(P, 1, \theta_{1}, y_{1}, A_{1}\right)$ and $T(P, k, u, \psi)=t\left(n, 1, \theta_{1}, \sigma_{1}, y_{1}, k, u, \psi\right)$. 


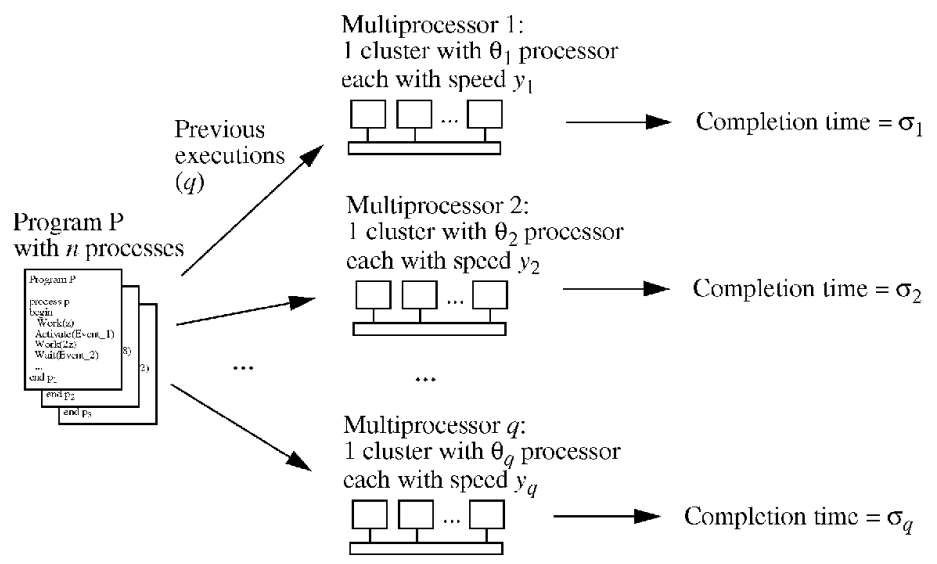

Fig. 5. The parameters $(n, q, \bar{\theta}, \bar{\sigma}$, and $\bar{y})$ describing the previous executions of the program.

TABLE 1

SUMMARY OF THE SYMBOLS USED IN THIS PAPER

\begin{tabular}{|c|c|}
\hline Notation & Description \\
\hline \multicolumn{2}{|c|}{ The symbols used for characterizing the parallel program } \\
\hline$n$ & The number of processors in the parallel program \\
\hline$q$ & The number of previous executions of the parallel program \\
\hline $\bar{\sigma}=\sigma_{1}, \ldots, \sigma_{q}$ & $\begin{array}{l}\text { The completion time vector, } \sigma_{i} \text { denotes the completion time in } \\
\text { previous execution number } i(1 \leq i \leq q)\end{array}$ \\
\hline$\theta=\theta_{1}, \ldots, \theta_{q}$ & $\begin{array}{l}\text { The multiprocessor size vector, } \theta_{i} \text { denotes the number of } \\
\text { processors in previous execution number } i(1 \leq i \leq q)\end{array}$ \\
\hline $\bar{y}=y_{1}, \ldots, y_{q}$ & $\begin{array}{l}\text { The multiprocessor speed vector, } y_{i} \text { denotes the speed of the } \\
\text { processors in previous execution number } i(1 \leq i \leq q)\end{array}$ \\
\hline \multicolumn{2}{|r|}{$\begin{array}{l}\text { The symbols used for characterizing the multiprocessor } \\
\text { for which we want to calculate the bound }\end{array}$} \\
\hline k & $\begin{array}{l}\text { The number of clusters in the multiprocessor for which we want } \\
\text { to calculate the bound }\end{array}$ \\
\hline$u$ & $\begin{array}{l}\text { The number of processors in each cluster in the multiprocessor } \\
\text { for which we want to calculate the bound }\end{array}$ \\
\hline$\psi$ & $\begin{array}{l}\text { The speed of the processors in the multiprocessor for which we } \\
\text { want to calculate the bound }\end{array}$ \\
\hline
\end{tabular}

Consequently, based on the information we have, the bound cannot be tighter than $t(n, q, \bar{\theta}, \bar{\sigma}, \bar{y}, k, u, \psi)$ when $q=$ 1. As $q$ increases, the set of programs corresponding to $n, q$, $\bar{\theta}, \bar{\sigma}$, and $\bar{y}$ becomes smaller, hence $t(n, q, \bar{\theta}, \bar{\sigma}, \bar{y}, k, u, \psi)$ becomes tighter in the sense that

$$
t(n, q, \bar{\theta}, \bar{\sigma}, \bar{y}, k, u, \psi) \leq t\left(n, 1, \theta_{1}, \sigma_{1}, y_{1}, k, u, \psi\right)
$$

when $q>1$. However, we cannot guarantee that $t(n, q, \bar{\theta}, \bar{\sigma}, \bar{y}, k, u, \psi)$ is optimal when $q>1$, i.e., using the information we have, it may be possible to obtain a bound which is somewhat tighter than $t(n, q, \bar{\theta}, \bar{\sigma}, \bar{y}, k, u, \psi)$ when $q>1$.

We also define a function

$$
s(q, \bar{\theta}, \bar{\sigma}, \bar{y}, k, u, \psi)=\max _{n} t(n, q, \bar{\theta}, \bar{\sigma}, \bar{y}, k, u, \psi) .
$$

Consequently, if we execute a program $P$ on a number of clusters and obtain the completion time vector $\bar{\sigma}$ (see Fig. 5), then $T(P, k, u, \psi) \leq s(q, \bar{\theta}, \bar{\sigma}, \bar{y}, k, u, \psi)$ independent of the number of processes in $P$. Again, the bound is optimal when $q=1$, i.e., for any $\varepsilon>0$, there is a program $P$ and a schedule $A_{1}$, such that $\sigma_{1}=T\left(P, 1, \theta_{1}, y_{1}, A_{1}\right)$ and $T(P, k, u, \psi)$ $\geq s\left(1, \theta_{1}, \sigma_{1}, y_{1}, k, u, \psi\right)-\varepsilon$. As $q$ increases $s(q, \bar{\theta}, \bar{\sigma}, \bar{y}, k, u, \psi)$ becomes tighter.

The fact that the bounds become tighter when $q$ increases is quite intuitive. Based on previous executions of the parallel program, we are able to bound the minimal completion time on a system with multiple clusters. If we have access to a large number of previous executions, i.e., if $q$ is large, we know more about the program and are thus able to obtain a tighter bound, i.e., $s(q, \bar{\theta}, \bar{\sigma}, \bar{y}, k, u, \psi)$ and $t(n, q, \bar{\theta}, \bar{\sigma}, \bar{y}, k, u, \psi)$ become tighter when $q$ increases.

\section{Evaluating a HeURISTic SCHEDULe}

Before we show how the functions $t(n, q, \bar{\theta}, \bar{\sigma}, \bar{y}, k, u, \psi)$ and $s(q, \bar{\theta}, \bar{\sigma}, \bar{y}, k, u, \psi)$ can be obtained, we will demonstrate the applicability of the bounds by evaluating a heuristic schedule of a parallel program.

The model for representing the execution behavior of the parallel program (see Fig. 4) is very general. The behavior of parallel programs using common synchronization techniques, 


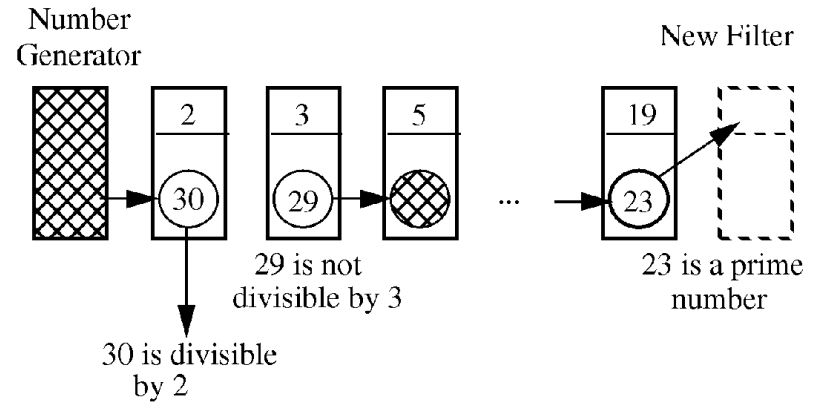

Fig. 6. The algorithm for generating prime numbers.

such as semaphores and monitors, can be represented with combinations of Waits and Activates. It has been shown that the behavior of parallel Ada programs and multithreaded Sun Solaris programs can be represented using this model [11], [14], as long as the length and the order of the sequential segments are independent of the way the processes are scheduled. This is the case for a number of parallel programs, e.g., most parallel programs for sorting, matrix manipulation, and the prime sieve program discussed below.

We are now going to show how $t(n, q, \bar{\theta}, \bar{\sigma}, \bar{y}, k, u, \psi)$ can be applied to a real case by bounding the completion time of a parallel implementation of an algorithm for generating all prime numbers smaller than or equal to a number $\mathrm{X}$. The algorithm generates the prime numbers in increasing order starting with prime number 2 and ending with the largest prime number smaller than or equal to $X$.

Fig. 6 shows how the algorithm works: There are a number of filters which form a line with a number generator feeding numbers into the line. Each filter filters out numbers which are divisible by its prime number, e.g., the first one filters out all even numbers. If a filter cannot divide a number, that number is forwarded to the next filter. Therefore, prime numbers will reach the end of the line. When a prime number reaches the end of the line, a new filter is created. In this case, the number generator stops by generating the number $3,000,000$.

The filter chain is cut up into contiguous subchains containing 500 filters each. Each such subchain is executed by a Sun Solaris thread, thus making the parallelism in the program relatively coarse grained. The maximum number of such threads, which is reached at the end of the execution, is 44 .

The program was first executed four times on a Sun Sparc Center 1000 using 1, 2, 4, and 8 processors, respectively. During these executions, the Solaris operating system was free to relocate threads from one processor to another. Based on these four executions, we got four completion time values $\sigma_{1}=5,523$ seconds $=92.0$ minutes, $\sigma_{2}=2,849$ seconds $=47.5$ minutes, $\sigma_{3}=1,597$ seconds $=26.5$ minutes, and $\sigma_{4}=979$ seconds $=16.5$ minutes (see Fig. 7a) corresponding to $\theta_{1}=1, \theta_{2}=2, \theta_{3}=4, \theta_{4}=8$, and $y_{1}=y_{2}=y_{3}=y_{4}$ $=136 * 10^{6}$ instructions per second, i.e., the processor speed of each processors in the Sun Sparc Center 1000 is 136 MIPS [17]. Based on these inputs we calculate

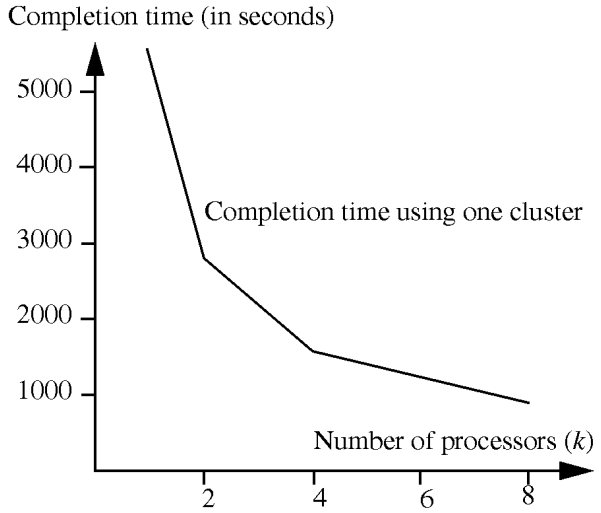

(a)

Completion time (in seconds)

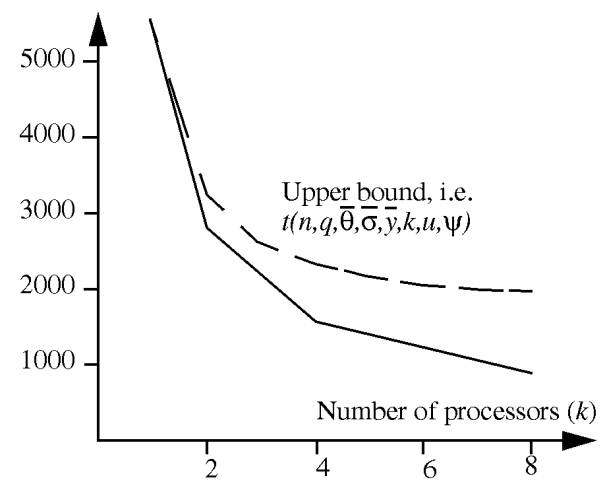

(b)

Completion time (in seconds)

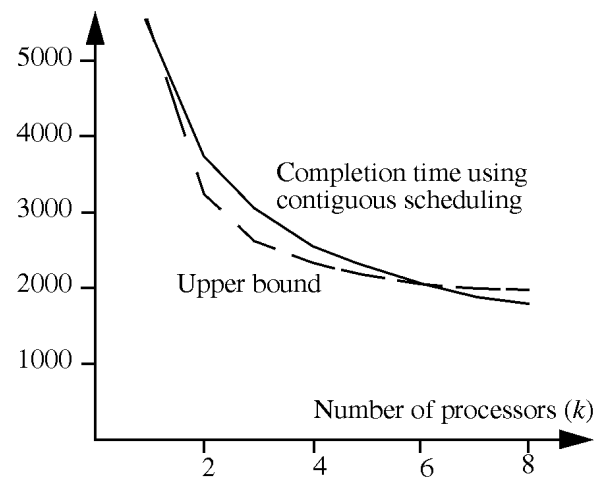

(c)

Fig. 7. Comparing the completion using contiguous scheduling with the upper bound.

$$
\begin{aligned}
& t(n, q, \bar{\theta}, \bar{\sigma}, \bar{y}, k, u, \psi)= \\
& t(44,4,\{1,2,4,8\},\{5,523,2,849,1,597,919\}, \\
& \left\{136 * 10^{6}, 136 * 10^{6}, 136 * 10^{6}, 136 * 10^{6}\right\}, \\
& \left.k, 1,136 * 10^{6}\right),
\end{aligned}
$$

i.e., in order not to complicate this example we only consider systems where $u=1, \psi=y_{1}=y_{2}=y_{3}=y_{4}=136 * 10^{6}$ instructions per second. Fig. $7 \mathrm{~b}$ shows the bound in the interval $(1 \leq k \leq 8)$. 
Solaris threads can be bound to processors using a routine called processor_bind (see [16] and [18]). Using our terminology, the Sun Sparc Center 1000 is a system with one cluster. However, by binding threads to processors, we are able to simulate a system with $k$ clusters of unit size, i.e., each processor becomes a cluster.

Using the processor_bind routine, a simple schedule for a system with $k$ clusters of unit size was implemented. The first $\lfloor n / k\rfloor$ threads are executed by processor one (thread one is the first filter subchain, thread two is the second filter subchain, and so on). Similarly, the next $\lfloor n / k\rfloor$ threads are executed by processor two. The last $n-(k-1) \times\lfloor n / k\rfloor$ threads are executed by processor $k$. We refer to this as contiguous scheduling, since the chain of threads is cut up into $k$ contiguous subchains, and each subchain is scheduled to a cluster.

Fig. 7c shows the completion time using contiguous scheduling and the upper bound. The figure shows that the completion time using contiguous scheduling is above the upper bound when the number of clusters $(k)$ is less than six. Consequently, in this interval we know that contiguous scheduling is not optimal for systems with $k$ clusters containing one processor each, and it is, thus, useful to look for other schedules. When $k$ is greater than or equal to six, the completion time using contiguous scheduling is below the upper bound. In this interval, the bound is less useful; it only tells us that contiguous scheduling may be optimal in this interval.

If the number of threads was unknown, the function $s\left(4,\{1,2,4,8\},\{5,523,2,849,1,597,919\},\left\{136 * 10^{6}, 136 * 10^{6}\right.\right.$, $\left.136 * 10^{6}, 136 * 10^{6}\right\}, k, 1,136 * 10^{6}$ ) could have been used for bounding the minimal completion time.

\section{Overview of the Method Used for Obtaining $t(n, q, \theta, \bar{\sigma}, \bar{y}, k, u, \psi)$ AND $s(q, \theta, \bar{\sigma}, \bar{y}, k, u, \psi)$}

This section gives a short overview of the detailed discussions in Sections 5, 6, and 7.

In order to obtain

$$
t(n, q, \bar{\theta}, \bar{\sigma}, \bar{y}, k, u, \psi)
$$

and

$$
s(q, \bar{\theta}, \bar{\sigma}, \bar{y}, k, u, \psi)
$$

we start with an arbitrary program $P$ with $n$ processes for which there exists schedules $A_{1}, \ldots, A_{q}$, such that $\sigma i=$ $T\left(P, 1, \theta_{i}, y_{i}, A_{i}\right)(1 \leq i \leq q) . P$ is then transformed into $q$ new programs $P_{i}^{\prime}(1 \leq i \leq q)$, each with $n$ processes, such that $\sigma_{i}=T\left(P_{i}^{\prime}, 1, \theta_{i}, y_{i}, A_{i}\right)$. The transformation guarantees that $T(P, k, u, \psi) \leq T\left(P^{\prime}, k, u, \psi\right)(1 \leq i \leq q)$. Each $P_{i}^{\prime}$ is transformed into a new program $P_{i}^{\prime \prime}$ with $n$ processes such that $\sigma_{i}=T\left(P_{i}^{\prime \prime}, 1, \theta_{i}, y_{i}, A_{i}\right)$. The transformation guarantees that $T\left(P^{\prime}, k, u, \psi\right) \leq T\left(P^{\prime \prime}, k, u, \psi\right)(1 \leq i \leq q)$. The transformation from $P_{i}^{\prime}$ into $P_{i}^{\prime \prime}$ is made in such a way that it is trivial to find a schedule resulting in $T\left(P_{i}^{\prime \prime}, k, u, \psi\right)$. Therefore, we are able to present a formula for $T\left(P_{i}^{\prime \prime}, k, u, \psi\right)$.
The transformations are such that, starting with a program $P$, we end up with $q$ programs $P_{i}^{\prime \prime}(1 \leq i \leq q)$. Therefore, if we consider the (infinite) set of all possible programs $\{P 1, P 2$, $P 3, \ldots\}$ with $n$ processes and such that $\sigma_{i}=T\left(P 1,1, \theta_{i}, y_{i}, A 1_{i}\right)=$ $T\left(P 2,1, \theta_{i}, y_{i} A 2_{i}\right)=T\left(P 3,1, \theta_{i}, y_{i}, A 3_{i}\right)=\ldots$ for some schedules $A 1_{i}, A 2_{i}, A 3_{i}, \ldots(1 \leq i \leq q)$, we end up with $q$ (infinite) sets of programs $\left\{P 1_{1}^{\prime \prime}, P 2_{1}^{\prime \prime}, P 3_{1}^{\prime \prime}, \ldots\right\},\left\{P 1_{2}^{\prime \prime}, P 2_{2}^{\prime \prime}, P 3_{2}^{\prime \prime}, \ldots\right\}, \ldots$, $\left\{P 1_{q}^{\prime \prime}, P 2_{q}^{\prime \prime}, P 3_{q}^{\prime \prime}, \ldots\right\}$. Using standard linear programming techniques, we will, from each of these $q$ sets, select a program $P X_{i}^{\prime \prime}$ such that $T\left(P X_{i}^{\prime \prime}, k, u, \psi\right)$ is maximal $(X=1$ or $X=2$ or $X=3$....), e.g., if $q=3$, we may select the programs $P 8_{1}^{\prime \prime}$, $P 1_{2}^{\prime \prime}$, and $P 6_{3}^{\prime \prime}$.

We know that, for each value $i(1 \leq i \leq q)$,

$$
T(P 1, k, u, \psi) \leq T\left(P X_{i}^{\prime \prime}, k, u, \psi\right)
$$

and

$$
T(P 2, k, u, \psi) \leq T\left(P X_{i}^{\prime \prime}, k, u, \psi\right)
$$

and

$$
T(P 3, k, u, \psi) \leq T\left(P X_{i}^{\prime \prime}, k, u, \psi\right) \ldots
$$

for all combinations of $k, u$, and $\psi$. The function $t(n, q, \bar{\theta}, \bar{\sigma}, \bar{y}, k, u, \psi)$ is therefore defined as the minimum $T\left(P X_{i}^{\prime \prime}, k, u, \psi\right)$ for all $i(1 \leq i \leq q)$. We also show that $t(n, q, \bar{\theta}, \bar{\sigma}, \bar{y}, k, u, \psi)$ is a nondecreasing function of $n$. Consequently, the function $s(q, \bar{\theta}, \bar{\sigma}, \bar{y}, k, u, \psi)$ is obtained by letting $n$ approach infinity in the formula for $t(n, q, \bar{\theta}, \bar{\sigma}, \bar{y}, k, u, \psi)$.

In order to obtain the bounds $t(n, q, \bar{\theta}, \bar{\sigma}, \bar{y}, k, u, \psi)$ and $s(q, \bar{\theta}, \bar{\sigma}, \bar{y}, k, u, \psi)$, we use a number of program transformations. However, the bounds are valid for all programs, i.e., the program for which we want to bound the completion time do not have to be transformed. For instance, the prime sieve program considered in the previous section was not transformed in any way. Nevertheless, we were able to bound the completion time of that program.

\section{Program Transformations}

Given a set of input values $n, q, \bar{\theta}, \bar{\sigma}, \bar{y}, k, u$ and $\psi$, we select an arbitrary program $P$ with $n$ processes for which there exists schedules $A_{1}, \ldots, A_{q}$, such that $\sigma_{i}=T\left(P, 1, \theta_{i}, y_{i}, A_{i}\right)$ $(1 \leq i \leq q)$. We then consider $q$ multiprocessors with one cluster each. The cluster in multiprocessor $i$ contains $\theta_{i}$ processors. The processor speed of multiprocessor $i$ is denoted $y_{i}$. Using multiprocessor $i$, the execution of $P$, using schedule $A_{i}$, is partitioned into $m_{i}$ equally sized time slots in such a way that all process synchronizations, terminations, and switches occur at the end of a time slot.

In order to obtain the new programs $P_{i}^{\prime}(1 \leq i \leq q)$, we add new synchronizations at the end of each time slot. These synchronizations guarantee that no processing done in slot $r\left(1<r \leq m_{i}\right)$ can be done unless all processing in slot $r-1$ has been completed. The net effect of this is that 

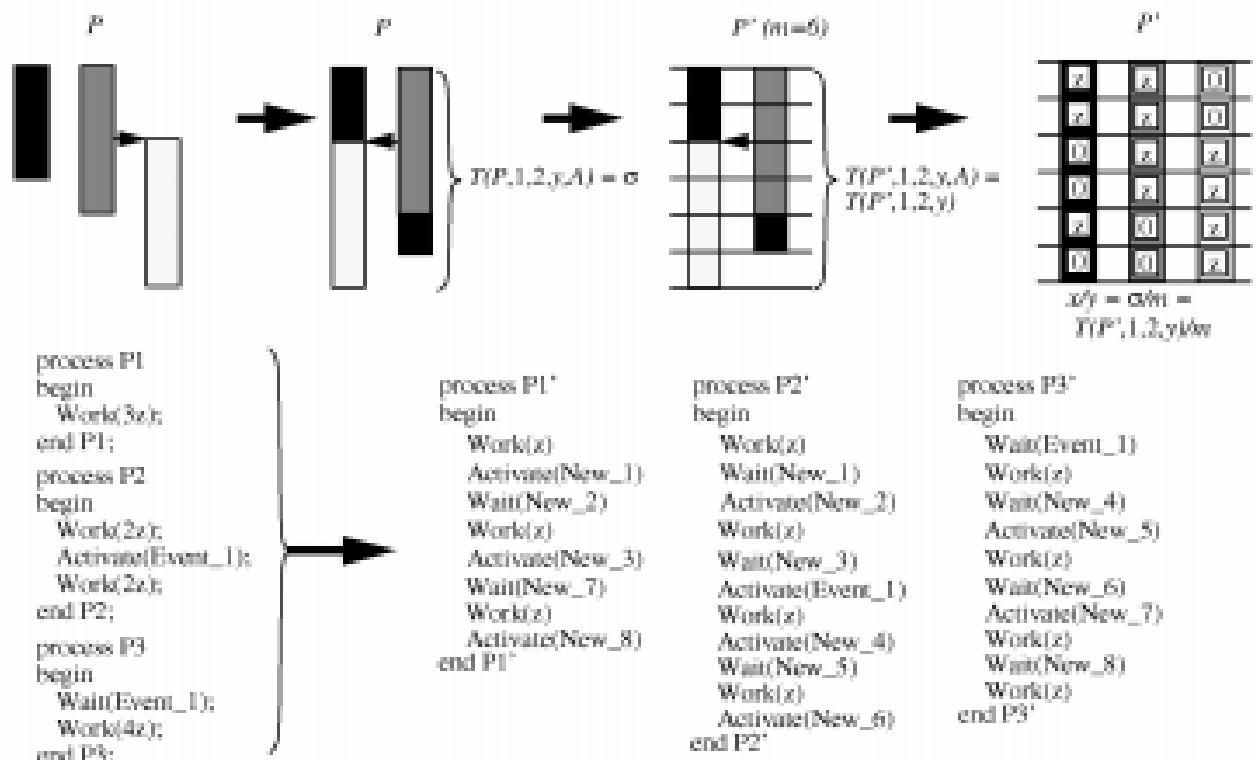

Fig. 8. Transforming program $P$ into a new program $P^{\prime}$.

a barrier synchronization is introduced at the end of each slot. Fig. 8 shows how a program $P$ is transformed into a new program $P^{\prime}$ using a multiprocessor with one cluster containing two processors. The synchronizations in each $P_{i}^{\prime}$ form a superset of the synchronizations in $P$. Consequently, $T(P, k, u, \psi) \leq T\left(P_{i}^{\prime}, k, u, \psi\right)(1 \leq i \leq q)$. However, from the definition of $P_{i}^{\prime}$ it is clear that

$$
\begin{aligned}
\sigma_{i} & =T\left(P, 1, \theta_{i}, y_{i}, A_{i}\right) \\
& =T\left(P_{i}^{\prime}, 1, \theta_{i}, y_{i}, A_{i}\right) \\
& =T\left(P_{i}^{\prime}, 1, \theta_{i}, y_{i}\right)(1 \leq i \leq q) .
\end{aligned}
$$

In order to simplify the discussion, we introduce an equivalent representation of each $P_{i}^{\prime}$ (see Fig. 8). Each process is represented as a vector of length $m_{i}$, i.e., the program is represented as $n$ vectors. In some situations, we will treat the vectors as one $m_{i} \times n$ matrix, where each column corresponds to a vector and each row to a time slot. $P_{i}^{\prime}(r, b)$ denotes entry $r$ in vector $b$, i.e., row $r$ in column $b$. The entries in $P_{i}^{\prime}$ can be either 0 or $z_{i}$. If a process is blocked in a certain time slot the corresponding entry in the corresponding vector is zero, otherwise the entry is $z_{i}$. The value $z_{i} / y_{i}$ denotes the execution time of a time slot using processors of speed $y_{i}$, i.e., $m_{i} * z_{i} / y_{i}=\sigma_{i}$. Consequently,

$$
z_{i}=\sigma_{i} * y_{i} / m_{i}=T\left(P_{i}^{\prime}, 1, \theta_{i}, y_{i}\right) * y_{i} / m_{i} .
$$

The total amount of work $(Z)$ performed by the program is unaffected by this transformation.

Let $A(b)(1 \leq b \leq n)$ denote the identity of the cluster where process $b$ is scheduled using schedule $A$, e.g., the schedule used in the rightmost part of Fig. 4 has the following entries: $A(1)=1, A(2)=1$, and $A(3)=2$. The execution time of row $r$ using schedule $A$ and a multiprocessor with $k$ clusters with $u$ processors each with processors speed $\psi$ can be calculated as:

$$
\begin{array}{r}
\max \left(\left(z_{i} / \psi\right), \max _{j=1}^{k}\left(\sum_{b=1}^{n}[A(b)=j] P_{i}^{\prime}(r, b)\right) /(u \Psi)\right)= \\
\max \left(z_{i}, \underset{j=1}{k}\left(\sum_{b=1}^{n}[A(b)=j] P_{i}^{\prime}(r, b)\right) / u\right) / \Psi,
\end{array}
$$

where the function $[A(b)=j]$ equals one if the conditions in brackets is true, otherwise $[A(b)=j]$ equals zero.

The execution time or row $r$ cannot be less than $z_{i} / \psi$. If the number of active processes scheduled to some cluster $j$ exceeds $u$ in time slot $r$, the execution time of slot $r$ exceeds $z_{i} / \psi$. In that case, the execution time of slot $r$ is the maximum execution time for the $k$ clusters. The execution time for cluster $j$ is

$$
\sum_{b=1}^{n}[A(b)=j] P_{i}^{\prime}(r, b) /(u \Psi) .
$$

In order to transform $P_{i}^{\prime}$ to $P_{i}^{\prime \prime}$, we start by creating $n$ ! copies of $P_{i}^{\prime}$. Each entry in each copy is divided by $n !$. The vectors in each copy are reordered so that each copy corresponds to one of the $n$ ! possible permutations of $n$ vectors. Vector number $b(1 \leq b \leq n)$ in copy number $c(1 \leq c<n !)$ is concatenated with vector number $b$ in copy $c+1$, thus forming a new program $P_{i}^{\prime \prime}$ with $n$ vectors of length $n ! * m_{i}$ (see Fig. 9). The execution time from slot $1+(c-1) * m_{i}$ to $c^{*} m_{i}(1 \leq c \leq n !)$, using $k$ clusters containing $u$ processors each, cannot be less than $T\left(P_{i}^{\prime}, k, u, \psi\right) / n$ !. Consequently, $T\left(P_{i}^{\prime}, k, u, \psi\right) \geq T\left(P_{i}^{\prime}, k, u, \psi\right)$. Obviously,

$$
T\left(P_{i}^{\prime \prime}, 1, \theta_{i}, y_{i}\right)=T\left(P_{i}^{\prime}, 1, \theta_{i}, y_{i}\right) .
$$

The total amount of work $(Z)$ performed by the program is unaffected by this transformation.

$T\left(P_{i}^{\prime \prime}, k, u, \psi, A\right)$ is not affected by the identity of the processes scheduled to different clusters, because if process $p_{1}$ is 


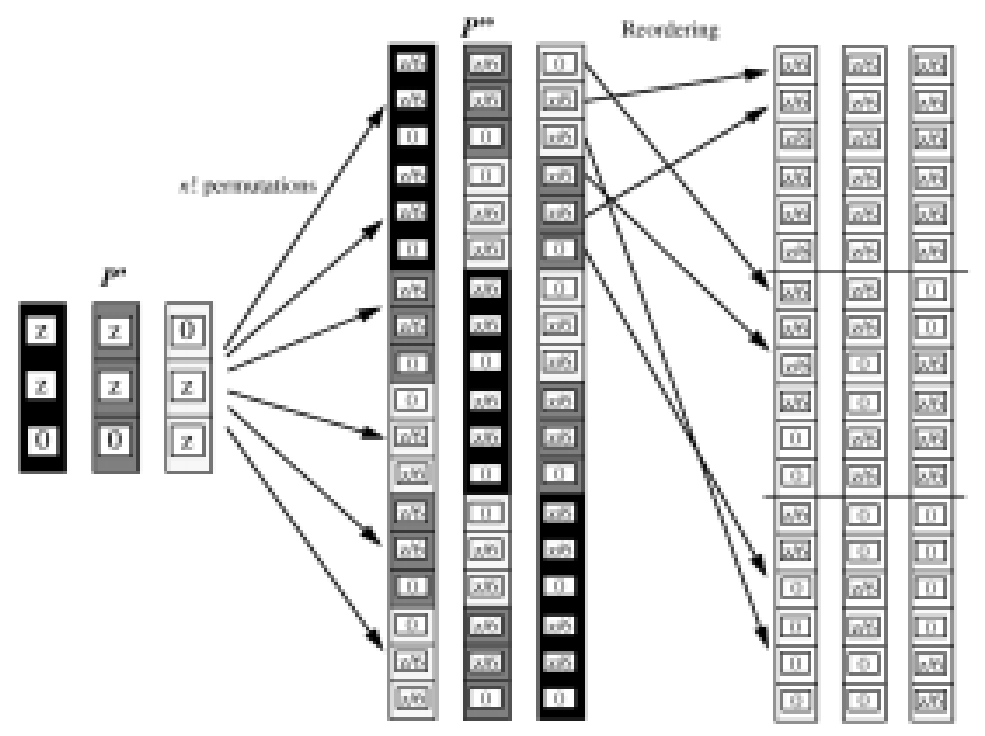

Fig. 9. Transforming a program $P^{\prime}$ into a new program $P^{\prime \prime}$. In this case, $n=3$, i.e., $n !=6$.

scheduled to cluster $C$ and process $p_{2}$ to cluster $B$ using schedule $A$, moving $p_{1}$ to $B$ and $p_{2}$ to $C$ is equivalent to reordering the rows in $P_{i}^{\prime \prime}$. Obviously, reordering the rows does not affect the completion time. Consequently, the completion time of $P_{i}^{\prime \prime}$ is affected only by the number of processes scheduled on the different clusters.

THEOREM 1. Consider a program $P_{i}^{\prime \prime}$. If there are $n_{1}$ vectors scheduled to cluster $C$ and $n_{2}$ vectors to cluster $B$ and $n_{1}<n_{2}$, the completion time cannot increase if we move one vector from cluster B to cluster $C$.

ProOF. We order the vectors in $P_{i}^{\prime \prime}$ in such a way that vectors 1 to $n_{1}$ are scheduled to cluster $C$ and vectors $n_{1}+1$ to $n_{1}+n_{2}$ are scheduled to cluster $B$. We then prove that moving vector number $n_{1}+1$ to cluster $C$ does not increase the completion time.

If vector $n_{1}+1$ has a zero in slot $r\left(1 \leq r \leq n !{ }^{*} m\right)$, the contribution to the completion time from row $r$ will not be affected by moving vector $n_{1}+1$ from cluster $B$ to cluster $C$. Consequently, we only have to consider rows for which the corresponding slot is $z / n$ ! in vector $n_{1}+1$. Moreover, if the number of nonzero slots in positions 1 to $n_{1}$ is smaller than the number of nonzero slots in positions $n_{1}+1$ to $n_{1}+n_{2}$, the contribution to the completion time from row $r$ does not increase.

From the way $P_{i}^{\prime \prime}$ has been obtained we know that, for each row $r$ such that position $n_{1}+1$ contains $z / n !$, and there are at least as many nonzero values in positions 1 to $n_{1}$ as in positions $n_{1}+1$ to $n_{1}+n_{2}$, there exists an $\left(n_{1}, n_{2}\right)$-permutation $r^{\prime}$. An $\left(n_{1}, n_{2}\right)$ permutation of a row is obtained by switching items $i$ and $n_{1}+n_{2}+1-i\left(1 \leq i \leq n_{1}\right)$ (see Fig. 10).

If the contribution to the completion time from row $r$ increases from $h(z / n !) /(u * \mathrm{y})$ to $(h+1)(z / n !) /\left(u^{*} \mathrm{y}\right)$ when we move vector $n_{1}+1$ from cluster $B$ to cluster $C$, there must be $h$ nonzero slots in positions 1 to $n_{1}$ in row $r$. In that case, we know that the symmetry of the $\left(n_{1}, n_{2}\right)$-permutation guarantees that there are at least $h+1$ nonzero slots in positions $n_{1}+1$ to $n_{1}+n_{2}$ in row $r^{\prime}$. Positions $n_{1}+n_{2}+1$ to $n$ are identical in $r$ and $r^{\prime}$. Therefore, the contribution to the completion time from $r^{\prime}$ will decrease with $(z / n !) /\left(u{ }^{*} \mathrm{y}\right)$ when moving vector $n_{1}+1$ from cluster $B$ to cluster $C$. Consequently, the completion time of $P_{i}^{\prime \prime}$ cannot increase if we move one vector from cluster $B$ to cluster $C$.

COROLlary. A schedule of $P^{\prime \prime}$ resulting in $T\left(P^{\prime \prime}, k, u, \psi\right)$ is obtained by first ordering the $n$ vectors in some arbitrary order, and then scheduling vector number $c+i * k$ to cluster $c(1 \leq c \leq k, 0 \leq i \leq\lfloor n / k\rfloor)$.

\section{Parallel Profile Vectors}

We now introduce the concept of a parallel profile vector $\bar{v}$. There is one parallel profile vector $\bar{v}$ for each program $P_{i}^{\prime \prime}$. Different programs may, however, correspond to the same parallel profile vector. Entry $x$ in $\bar{v}(1 \leq x \leq \theta)$ contains the fraction of the rows in the matrix corresponding to $P_{i}^{\prime \prime}$ where there are $x$ nonzero slots. The $P_{i}^{\prime \prime}$ program corresponding to the $P^{\prime}$ program in Fig. 9 has the following parallel profile vector $(\theta=2): \bar{v}(1)=1 / 6$, i.e., $n$ ! of the $6 n$ ! rows in $P_{i}^{\prime \prime}$ contain one nonzero slot, and $\bar{v}(2)=5 / 6$, i.e., $5 n$ ! of the rows in $P_{i}^{\prime \prime}$ contain two nonzero slots.

The transformations are such that starting with a program $P$ we end up with $q$ programs $P_{i}^{\prime \prime}(1 \leq i \leq q)$. Therefore, if we consider the (infinite) set of all possible programs $\{P 1, P 2, P 3, \ldots\}$ with $n$ processes and such that $\sigma_{i}=T(P 1,1$, $\left.\theta_{i}, y_{i}, A 1_{i}\right)=T\left(P 2,1, \theta_{i}, y_{i}, A 2_{i}\right)=T\left(P 3,1, \theta_{i}, y_{i}, A 3_{i}\right)=\ldots$ for some 
(2,4)-permutation

\begin{tabular}{|l|c|c|c|c|c|c|c|c|c|c|}
\hline$z / n !$ & 0 & $z / n !$ & 0 & 0 & 0 & $z / n !$ & $z / n !$ & 0 & 0 & $z / n !$ \\
\hline 0 & 0 & $z / n !$ & 0 & 0 & $z / n !$ & $z / n !$ & $z / n !$ & 0 & 0 & $z / n !$ \\
\hline
\end{tabular}

(2,3)-permutation

\begin{tabular}{|l|c|c|c|c|c|c|c|c|c|c|}
\hline$z / n !$ & 0 & $z / n !$ & 0 & 0 & 0 & $z / n !$ & $z / n !$ & 0 & 0 & $z / n !$ \\
\hline 0 & 0 & $z / n !$ & 0 & $z / n !$ & 0 & $z / n !$ & $z / n !$ & 0 & 0 & $z / n !$ \\
\hline
\end{tabular}

Fig. 10. One (2, 4)-permutation and one (2, 3)-permutation of the same row.

schedules $A 1_{i}, A 2_{i}, A 3_{i}, \ldots(1 \leq i \leq q)$, we end up with $q$ (infinite) sets of programs $\left\{P 1_{1}^{\prime \prime}, P 2_{1}^{\prime \prime}, P 3_{1}^{\prime \prime}, \ldots\right\},\left\{P 1_{2}^{\prime \prime}, P 2_{2}^{\prime \prime}, P 3_{2}^{\prime \prime}, \ldots\right\}, \ldots$, $\left\{P 1_{q}^{\prime \prime}, P 2_{q}^{\prime \prime}, P 3_{q}^{\prime \prime}, \ldots\right\}$.

THEOREM 2. Consider two programs $P X_{i}^{\prime \prime}$ and $P Y_{i}^{\prime \prime}$ in a the set $\left\{P 1_{i}^{\prime \prime}, P 2_{i}^{\prime \prime}, P 3_{i}^{\prime \prime}, \ldots\right\}$, such that the parallel profile vectors are the same for both programs. In that case, $T\left(P X_{i}^{\prime \prime}, k, u, y\right)=T\left(P Y_{i}^{\prime \prime}, k, u, y\right)$ for all combinations of $k, u$, and $y$.

PROOF. We consider two exhaustive cases. First, the case when the number of rows is the same for $P X_{i}^{\prime \prime}$ and $P Y_{i}^{\prime \prime}$ and, then, the case when the number rows are different.

If the number of rows is the same, the only possible difference between $P X_{i}^{\prime \prime}$ and $P Y_{i}^{\prime \prime}$ is the order in which the rows appear. Reordering the rows does not affect the minimal completion time.

If there are more rows in one program, say $P Y_{i}^{\prime \prime}$, the transformations guarantee that if $P X_{i}^{\prime \prime}$ contains $m^{*} n$ ! rows, then $P Y_{i}^{\prime \prime}$ contains $r^{*} m^{*} n$ ! rows $(r>1)$. The transformations also guarantee that, if there are $f$ identical copies of a row with a certain combination of zero and nonzero values in $P X_{i}^{\prime \prime}$, there are $r * f$ copies of the same combination of zero and nonzero values in $P Y_{i}^{\prime \prime}$. However, compared to $P X_{i}^{\prime \prime}$, each entry in $P Y_{i}^{\prime \prime}$ has been divided with $r$, i.e., each row in $P Y_{i}^{\prime \prime}$ has an execution time which is $r$ times shorter than the corresponding row in $P X_{i}^{\prime \prime}$. Consequently, $T\left(P X_{i}^{\prime \prime}, k, u, y\right)=T\left(P Y_{i}^{\prime \prime}, k, u, y\right)$ for all combinations of $k, u$, and $y$.

Consequently, the only difference between two programs in a the set $\left\{P 1_{i}^{\prime \prime}, P 2_{i}^{\prime \prime}, P 3_{i}^{\prime \prime}, \ldots\right\}$ which is relevant in this context is the difference between their parallel profile vectors. We want to find programs $P_{i}^{\prime \prime}$ such that $T\left(P_{i}^{\prime \prime}, k, u, \psi\right)$ is maximal. In order to find these programs, we will calculate their corresponding parallel profile vectors.

As shown in the rightmost part of Fig. 9, the rows in a $P^{\prime \prime}$ program can be partitioned into $\theta_{i}$ groups, such that all rows in the same group contain the same number of nonzero slots (some groups may be empty). If the total number of rows in $P_{i}^{\prime \prime}$ is $m_{i}{ }^{*} n !$, the number of rows in group $x$, i.e., the group where each row contains $x\left(1 \leq x \leq \theta_{i}\right)$ nonzero slots, is $\bar{v}_{i}(x) * m_{i} * n$ !. We know that, in group $x$, all possible combinations of $n-x$ zero and $x$ nonzero slots occur equally frequently. Consequently, the execution time for group $x$, using the schedule in the corollary to Theorem 1 and a multiprocessor with $k$ clusters containing $u$ processors of speed $\psi$ each, can be explicitly calculated by simply generating the rows and calculating the execution time of each row. However, this is extremely inefficient, making it virtually impossible to handle reasonable parameters.

If the nonzero entries in the matrix corresponding to $P_{i}^{\prime \prime}$ equal $z_{i} / n$ !, the execution time of group $x$ in $P_{i}^{\prime \prime}$ using the schedule in the corollary to Theorem 1 and a multiprocessor with $k$ clusters containing $u$ processors of speed $\psi$ each is obviously $\bar{v}_{i}(x) * m_{i} * T\left(P x_{i}^{\prime \prime}, k, u, \psi\right)$, where $P_{i}^{\prime \prime}$ contains $n$ ! rows, each corresponding to one of the possible permutations of $n-x$ zeros and $x$ values $z_{i} / n$ !. Consequently, the execution time for group $x$ can be expressed as

$$
\begin{gathered}
\bar{v}_{i}(x) * m_{i} * n ! *\left(z_{i} / n !\right) * g(n, k, u, x) / \psi= \\
\bar{v}_{i}(x) * \sigma_{i} * y_{i} * g(n, k, u, x) / \psi,
\end{gathered}
$$

where $g(n, k, u, x)=T\left(P x_{i}^{\prime \prime}, k, u,\left(z_{i} / n !\right)\right) / n$ !, for a program $P x_{i}^{\prime \prime}$ which contains $n$ ! rows each corresponding to one of the possible permutations of $n-x$ zeros and $x$ values $z_{i} / n !$. The reason for this is that,

$$
\begin{aligned}
& \bar{v}_{i}(x) * m_{i} * n ! *\left(z_{i} / n !\right) * g(n, k, u, x) / \psi \\
& =\bar{v}_{i}(x) * m_{i} *\left(z_{i} / n !\right) * T\left(P x_{i}^{\prime \prime}, k, u,\left(z_{i} / n !\right)\right) / \psi \\
& =\bar{v}_{i}(x) * m_{i} * T\left(P x_{i}^{\prime \prime}, k, u, \psi\right),
\end{aligned}
$$

for a program $P x_{i}^{\prime \prime}$ which contains $n$ ! rows each corresponding to one of the possible permutations of $n-x$ zeros and $x$ values $z_{i} / n$ !. The last equality follows from the fact that $T(P$, $\left.k, u, y_{1}\right) / y_{2}=T\left(P, k, u, y_{2}\right) / y_{1}$ (see Section 2). The formula for calculating $g(n, k, u, x)$ is shown in Equation 1 (see also Appendix A on http://www.ide.hk-r.se/ lasse/tpds, the formula for the function $g(n, k)=g(n, k, 1, k)$ has been presented previously [9]). Fig. 11 shows $g(100,20,1, x)$ in the interval $1 \leq x \leq$ 40. Note that the function is convex in some intervals and concave in others.

The decreasing sequences used in the function $\pi$ in Equation 1 can be generated by the algorithm below, where the least decreasing sequence of length $\mu$ and sum $\alpha$ is the sequence $\{[\alpha / \mu\rceil, \ldots,\lceil\alpha / \mu\rceil,\lfloor\alpha / \mu\rfloor, \ldots,\lfloor\alpha / \mu]\}$. If $\alpha_{\mu}$ is the remainder when $\alpha$ is divided by $\mu$, the number of $[\alpha / \mu\rceil$ : s is $\alpha_{\mu}$, and the number of $\lfloor\alpha / \mu\rfloor: \mathrm{s}$ is $\mu-\alpha_{\mu}$ making the sum of the sequence $\alpha$.

Algorithm for generating decreasing sequences I of length $\mu$ and sum $\alpha$, and for which $i_{1}=\lambda$ :

1) Take $I$ as $\left(\lambda, I^{\prime}\right)$, where $I^{\prime}$ is the least decreasing sequence of length $\mu-1$ and sum $\alpha-1$. 


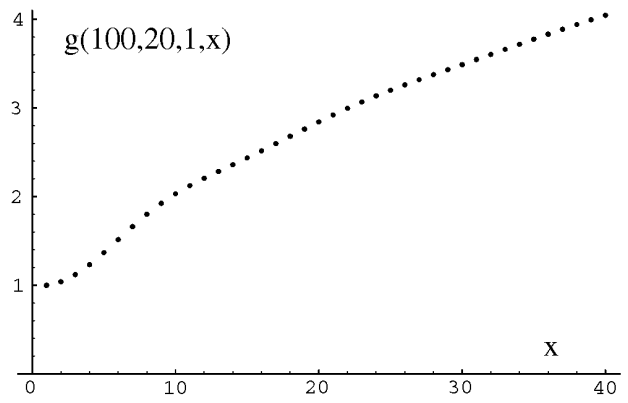

Fig. 11. The function $g(100,20,1, x),(1 \leq x \leq 40)$.

2) Find the rightmost position in $I$, say $j_{1}$, which fulfills:

a) $1<j_{1}<\mu$

b) $i_{j_{1}}<\lambda$

c) $i_{j_{1}}<i_{j_{1}-1}$ or $j_{1}=2$

d) $i_{j_{1}+1}>0$

The algorithm terminates if no such $j_{1}$ can be found.

3) The next sequence is obtained from $I$ by increasing the entry in position $j_{1}$ by one and replacing the subsequence $\left\{i_{j_{1}+1}, \ldots, i_{\mu}\right\}$ with the least decreasing subsequence of length $\mu-j_{1}$ and sum $\sum_{j=j_{1}+1}^{\mu} i_{j}-1$.

4) Go to Step 2.

This algorithm generates all decreasing sequences $I$, and each sequence is generated exactly once. The proof for this has been presented previously ([9]).

\section{Defining $t(n, q, \bar{\theta}, \bar{\sigma}, \bar{y}, k, u, \psi)$ AND $s(q, \theta, \bar{\sigma}, \bar{y}, k, u, \psi)$}

Using $g(n, k, u, x)$, the minimum completion time, i.e., the completion time using the schedule in the corollary to Theorem 1, can be expressed as:

$$
T\left(P_{i}^{\prime \prime}, k, u, \psi\right)=\sum_{x=1}^{\Theta_{i}} \bar{v}_{i}(x) * \sigma_{i} * y_{i} * g(n, k, u, x) / \psi .
$$

We want to find the $\bar{v}_{i}$ which maximizes this sum. However, there are restrictions. First, there are two obvious restrictions on $\bar{v}_{i}: \sum_{x=1}^{\Theta_{i}} \bar{v}_{i}(x)=1$ and $0 \leq \bar{v}_{i}(x)\left(1 \leq x \leq \theta_{i}\right)$. Moreover, $\sum_{x=1}^{\Theta_{i}} x \bar{v}_{i}(x) y_{i} \sigma_{i}$ denotes the total amount of work $(Z)$ performed by the program, i.e., $\sum_{x=1}^{\Theta_{i}} x \bar{v}_{i}(x) y_{i} \sigma_{i}=Z$. Obviously, $\mathrm{Z} \leq \min _{j} \theta j{ }^{*} s_{j}{ }^{*} y_{j}(1 \leq j \leq q)$. Therefore,

$$
\sum_{x=1}^{\Theta_{i}} x \bar{v}_{i}(x) y_{i} \sigma_{i} \leq \min _{j} \theta_{j} * \sigma_{j} * y_{j}(1 \leq j \leq q) .
$$

This means that we end up with the following optimization problem:

$$
\max _{\bar{v}_{i}} \sum_{x=1}^{\Theta_{i}} \bar{v}_{i}(x) * \sigma_{i} * y_{i} * g(n, k, u, x) / \psi
$$

under the restrictions:

$$
\begin{aligned}
& \sum_{x=1}^{\Theta_{i}} \bar{v}_{i}(x)=1, \bar{v}_{i}(x) \geq 0\left(1 \leq x \leq \theta_{i}\right), \\
& \sum_{x=1}^{\Theta_{i}} x \bar{v}_{i}(x) y_{i} \sigma_{i} \leq \min _{j} \theta_{j} * \sigma_{j} * y_{j}(1 \leq j \leq q) .
\end{aligned}
$$

This is an optimization problem which, for example, can be solved using the simplex method [1].

Consider a program $P$ with $n$ processes for which there exists schedules $A_{1}, \ldots, A_{q}$, such that $\sigma_{i}=T\left(P, 1, \theta_{i}, y_{i}, A_{i}\right)$. The transformations guarantee that for each value

$$
\begin{aligned}
& i(1 \leq i \leq q) T(P, k, u, \psi) \leq T\left(P_{i}^{\prime \prime}, k, u, \psi\right) \leq \\
& \max _{\bar{v}_{i}} \sum_{x=1}^{\Theta_{i}} \bar{v}_{i}(x) * \sigma_{i} * y_{i} * g(n, k, u, x) / \psi .
\end{aligned}
$$

$$
\begin{aligned}
& \text { If } n \leq k^{*} u \text { then } g(n, k, u, x)=1 \text {, otherwise } g(n, k, u, x) \text { is specified below, where } w=\lfloor n / k\rfloor \text { and } n_{k}=n-k w \text { : } \\
& \text { If } n_{k}=0 \text {, then } g(n, k, u, x)=\frac{1}{\left(\begin{array}{l}
n \\
x
\end{array}\right)} \sum_{l=1}^{\min (w, x)} \max \left(1, \frac{l}{u}\right) \pi(k, w, x, l) \quad \text {, otherwise } g(n, k, u, x)=
\end{aligned}
$$

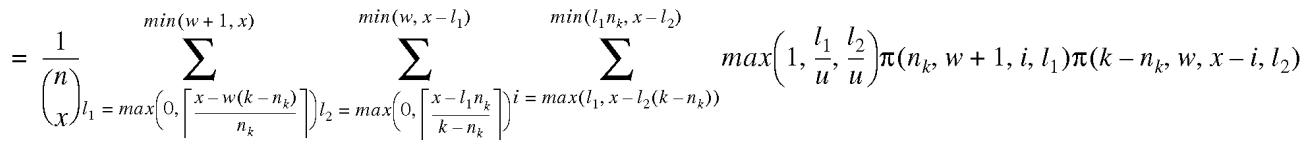

$$
\begin{aligned}
& \pi(k, w, x, l)=\sum_{I}\left(\begin{array}{l}
w \\
i_{1}
\end{array}\right) \cdot \ldots \cdot\left(\begin{array}{l}
w \\
i_{k}
\end{array}\right) \frac{k !}{b(I)} a(I, j) !
\end{aligned}
$$




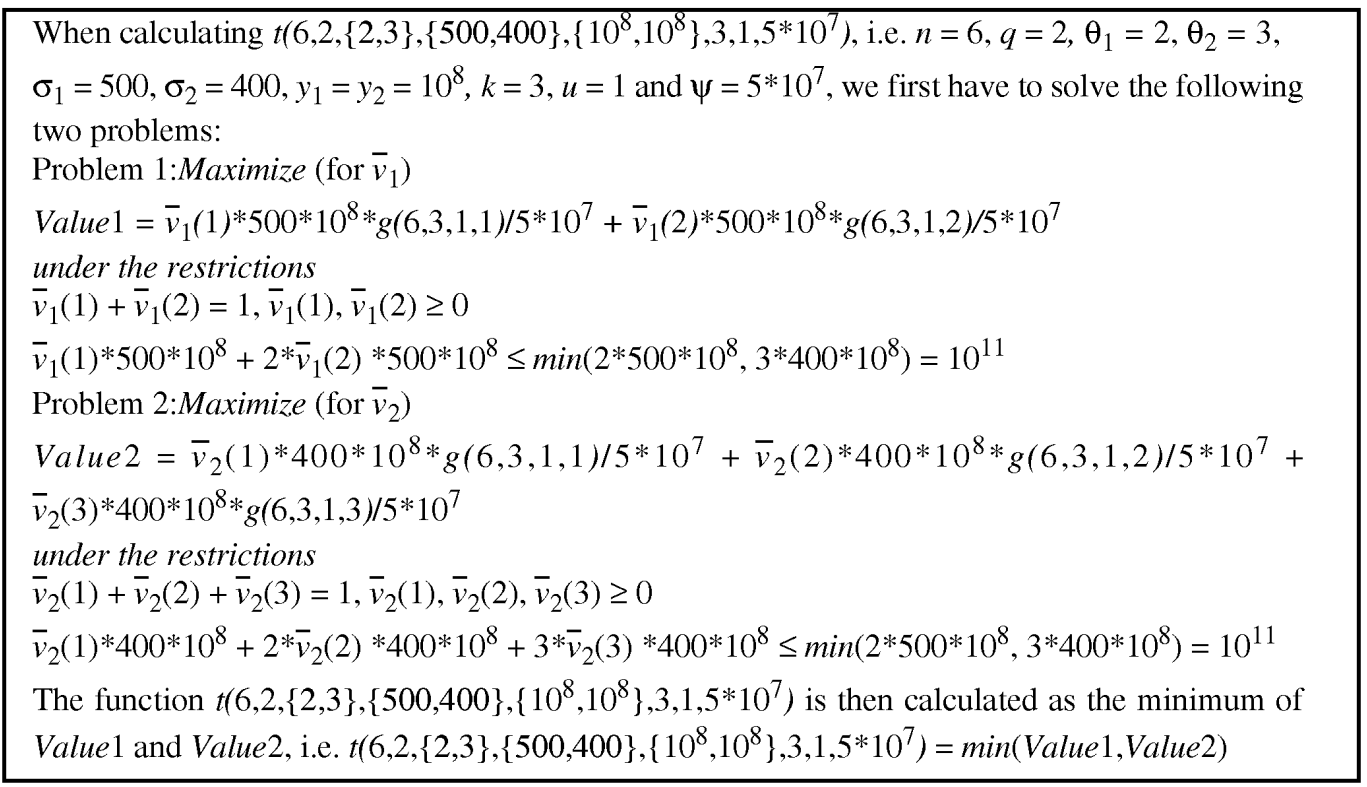

Example 1. Example showing how $t(n, q, \bar{\theta}, \bar{\sigma}, \bar{y}, k, u, \psi)$ is calculated.

The function $t(n, q, \bar{\theta}, \bar{\sigma}, \bar{y}, k, u, \psi)$ is, therefore, defined as:

$$
\min _{i} \max _{\bar{v}_{i}} \sum_{x=1}^{\Theta_{i}} \bar{v}_{i}(x) * \sigma_{i} * y_{i} * g(n, k, u, x) / \psi
$$

under the restrictions:

$$
\begin{aligned}
& \sum_{x=1}^{\Theta_{i}} \bar{v}_{i}(x)=1, \bar{v}_{i}(x) \geq 0\left(1 \leq x \leq \theta_{i}\right), \\
& \sum_{x=1}^{\Theta_{i}} x \bar{v}_{i}(x) y_{i} \sigma_{i} \leq \min _{j} \theta_{j} * \sigma_{j} * y_{j}(1 \leq j \leq q) .
\end{aligned}
$$

See also Example 1.

When $q=1$, the bound $t\left(n, 1, \theta_{1}, \sigma_{1}, y_{1}, k, u, \psi\right)$ is based on one program $P_{1}^{\prime \prime}$. From the transformations leading to $P_{1}^{\prime \prime}$, we know that $T(P, k, u, \psi)=T\left(P_{1}^{\prime \prime}, k, u, \psi\right)$ for all programs $P$ corresponding to $n, \theta_{1}, \sigma_{1}$, and $y_{1}$. However, the transformations also guarantee that $P_{1}^{\prime \prime}$ is one of the programs which correspond to $n, \theta_{1}, \sigma_{1}$, and $y_{1}$, i.e., it has $n$ processes, and there exists a schedule $A_{1}$, such that $\sigma_{1}=T\left(P_{1}^{\prime \prime}, 1, \theta_{1}, y_{1}, A_{1}\right)$. Consequently, when $q=1$ the bound is optimal because $t\left(n, 1, \theta_{1}, \sigma_{1}, y_{1}, k, u, \psi\right)=T\left(P_{1}^{\prime \prime}, k, u, \psi\right)$.

When $q$ grows, $t(n, q, \bar{\theta}, \bar{\sigma}, \bar{y}, k, u, \psi)$ decreases, i.e., the bound becomes tighter. There are two reasons for this. First, $\min _{j} \theta_{j}^{*} \sigma_{j}^{*} y_{j}(1 \leq i, j \leq q)$ decreases, i.e., the restriction

$$
\sum_{x=1}^{\Theta_{i}} x \bar{v}_{i}(x) y_{i} \sigma_{i} \leq \min _{j} \theta_{j} * \sigma_{j} * y_{j}(1 \leq i, j \leq q)
$$

disqualifies more programs, thus reducing

$$
\max _{\bar{v}_{i}} \sum_{x=1}^{\Theta_{i}} \bar{v}_{i}(x) * \sigma_{i} * y_{i} * g(n, k, u, x) / \psi .
$$

Second, there are more programs $P_{1}^{\prime \prime}$, thus reducing the minimum $T\left(P_{1}^{\prime \prime}, k, u, \psi\right)(1 \leq i \leq q)$.
Obviously, $t\left(n, 1, \theta_{1}, \sigma_{1}, y_{1}, k, u, \psi\right)$ is a nondecreasing function of $n$ for constant $\theta_{1}, \sigma_{1}, y_{1}, k, u$, and $\psi$ (one can always add a process which is blocked during the entire execution of the program). We know that

$$
t\left(n, 1, \theta_{1}, \sigma_{1}, y_{1}, k, u, \psi\right)=T\left(P_{1}^{\prime \prime}, k, u, \psi\right)
$$

for some program $P_{1}^{\prime \prime}$ such that, $\sigma_{1}=T\left(P_{1}^{\prime \prime}, 1, \theta_{1}, y_{1}, A_{1}\right)$ for some schedule $A_{1}$. We also know that

$$
T\left(P_{1}^{\prime \prime}, k, u, \psi\right)=\frac{\sigma_{1} y_{1}}{\psi} \sum_{x=1}^{\theta_{1}} v(x) g(n, k, u, x) .
$$

From the definition of $g(n, k, u, x)$, it is clear that $g(n, k, u, x)$ is a nondecreasing function of $x$ for constant $n, k$, and $u$. Consequently,

$$
\begin{aligned}
& t\left(n, 1, \theta_{1}, \sigma_{1}, y_{1}, k, u, \psi\right) \\
& \quad=T\left(P_{1}^{\prime \prime}, k, u, \psi\right) \\
& \quad=\sigma_{i} * y_{i} * g\left(n, k, u, \theta_{1}\right) / \psi,
\end{aligned}
$$

i.e., $g(n, k, u, x)$ is a nondecreasing function of $n$ for constant $k, u$, and $x$. Consequently, $G(k, u, x)=\max _{n} g(n, k, u, x)=$ $\lim _{n \rightarrow \infty} g(n, k, u, x)$. The formula for calculating the function $G(k, u, x)=g(n, k, u, x)$ is obtained by letting $n$ approach infinity in the formulas shown in Equation 1. The result of this is shown in Equation 2 (see also Appendix B on http://www.ide.hk-r.se/ lasse/tpds).

Consequently,

$$
\begin{aligned}
& s(q, \bar{\theta}, \bar{\sigma}, \bar{y}, k, u, \psi)=\max _{n} t(n, q, \bar{\theta}, \bar{\sigma}, \bar{y}, k, u, \psi)= \\
& \max _{n} \min _{i} \max _{\bar{v}_{i}} \sum_{x=1}^{\Theta_{i}} \bar{v}_{i}(x) * \sigma_{i} * y_{i} * g(n, k, u, x) / \psi
\end{aligned}
$$

under the restrictions:

$$
\sum_{x=1}^{\Theta_{i}} \bar{v}_{i}(x)=1, \bar{v}_{i}(x) \geq 0\left(1 \leq x \leq \theta_{i}\right)
$$




$$
G(k, u, x)=\frac{k ! x !}{k^{q} u} \sum_{t=1}^{x} \max (l, u) \sum_{I} \frac{1}{\prod_{j_{1}=1}^{k} i_{j_{1}} ! \prod_{j_{2}=1}^{b(I)} a\left(I, j_{2}\right) !} \text {, where the second sum is taken over all sequences of }
$$

nonnegative integers $I=\left\{i_{1}, \ldots, i_{k}\right\}$ which are decreasing, i.e. $i_{j} \geq i_{j+1}$ for all $j=1, \ldots, k-1$, and for which $i_{1}=l$ and $\sum_{j=1}^{k} i_{j}=x$. The functions $a(I, j)$ and $b(I)$ are defined in the following way:

$a(I, j)=$ the number of occurrences of the $j$ :th distinct integer in $I$.

$b(I)=$ the number of distinct integers in $I$.

Equation 2. The formula for $G(k, u, x)$.

$$
\sum_{x=1}^{\Theta_{i}} x \bar{v}_{i}(x) y_{i} \sigma_{i} \leq \min _{j} \theta_{j} * \sigma_{j} * y_{j}(1 \leq j \leq q) .
$$

The only part which is affected by $n$ is $g(n, k, u, x)$. Moreover, we know that $G(k, u, x)=\max _{n} g(n, k, u, x)$. Consequently, $s(q, \bar{\theta}, \bar{\sigma}, \bar{y}, k, u, \psi)=\min _{i} \max _{\bar{v}_{i}} \sum_{x=1}^{\Theta_{i}} \bar{v}_{i}(x) * \sigma_{i} * y_{i} * G(k, u, x) / \psi$ under the restrictions:

$$
\begin{aligned}
& \sum_{x=1}^{\Theta_{i}} \bar{v}_{i}(x)=1, \bar{v}_{i}(x) \geq 0\left(1 \leq x \leq \theta_{i}\right), \\
& \sum_{x=1}^{\Theta_{i}} x \bar{v}_{i}(x) y_{i} \sigma_{i} \leq \min _{j} \theta_{j} * \sigma_{j} * y_{j}(1 \leq j \leq q) .
\end{aligned}
$$

Again, this is an optimization problem which can be solved using the simplex method [1].

When $q=1$, the bound $s\left(1, \theta_{1}, \sigma_{1}, y_{1}, k, u, \psi\right)$ is optimal, because for any $\varepsilon>0$, there is a program $P_{1}^{\prime \prime}$ and a schedule $A_{1}$, such that $\sigma_{1}=T\left(P_{1}^{\prime \prime}, 1, \theta_{1}, y_{1}, A_{1}\right)$, and $T(P, k, u, \psi) \geq s(1$, $\left.\theta_{1}, \sigma_{1}, y_{1}, k, u, \psi\right)-\varepsilon$. The reason for this is that it is always possible to find a large enough $n$ such that

$$
\begin{gathered}
\max _{\bar{v}_{i}} \sum_{x=1}^{\Theta_{1}} \bar{v}_{1}(x) * \sigma_{1} * y_{1} * g(n, k, u, x) / \psi \geq \\
\max _{\bar{v}_{i}} \sum_{x=1}^{\Theta_{1}} \bar{v}_{1}(x) * \sigma_{1} * y_{1} * G(k, u, x) / \psi-\varepsilon .
\end{gathered}
$$

Consequently, if we consider a vector $\bar{v}_{1}$ such that

$$
\begin{aligned}
& s\left(1, \theta_{1}, \sigma_{1}, y_{1}, k, u, \psi\right)= \\
& \max _{\bar{v}_{1}} \sum_{x=1}^{\Theta_{1}} \bar{v}_{1}(x) * \sigma_{1} * y_{1} * G(k, u, x) / \psi,
\end{aligned}
$$

and select a large enough $n$, then there is a program $P_{1}^{\prime \prime}$ with $n$ processes and a schedule $A_{1}$, such that $\sigma_{1}=T\left(P_{1}^{\prime \prime}, 1\right.$, $\left.\theta_{1}, y_{1}, A_{1}\right)$ and

$$
\begin{aligned}
& T\left(P_{1}^{\prime \prime}, k, u, \psi\right)= \\
& \max _{\bar{v}_{1}} \sum_{x=1}^{\Theta_{1}} \bar{v}_{1}(x) * \sigma_{1} * y_{1} * g(n, k, u, x) / \psi \geq \\
& \max _{\bar{v}_{1}} \sum_{x=1}^{\Theta_{1}} \bar{v}_{1}(x) * \sigma_{1} * y_{1} * G(k, u, x) / \psi-\varepsilon= \\
& s\left(1, \theta_{1}, \sigma_{1}, y_{1}, k, u, \psi\right)-\varepsilon .
\end{aligned}
$$

\section{Discussion And Related Work}

The results are based on the assumption of zero synchronization overhead. Experience from commercial multiprocessor systems show that, due to poor cache performance, the overhead often grows with the number of processors in a cluster [10]. The reason for this is that when the number of processors is large, processes are often migrated from one processor to another and, in that case, the data associated with the migrated process has to be moved to the cache of the new processor. As processor caches get larger, the process migration penalty within a cluster will increase for all cluster sizes. However, run-time relocation is more frequent in large clusters (in unit-sized clusters there is no relocation at all). If the overhead is larger in the one-cluster systems used for obtaining the recorded information (see Fig. 5) than in the target multiprocessor (defined by $k, u$, and $\psi$ ), the bounds are still valid. They may, however, be somewhat less tight than they could have been.

Given a parallel program and a set of recordings from previous executions, the results can also be used for selecting a multiprocessor configuration with a guaranteed performance. For instance, if $q$ previous executions of a parallel program $P$ with $n$ processes have resulted in a completion time vector $\bar{\sigma}$ corresponding to the vectors $\bar{\theta}$ and $\bar{y}$ (see Fig. 5), we know that it is possible to find a schedule which has a completion time shorter than or equal to $t(n, q, \bar{\theta}, \bar{\sigma}, \bar{y}, k, u, \psi)$ for a multiprocessor with $k$ clusters containing $u$ processors with speed $\psi$. This information can be very useful when selecting a multiprocessor configuration for a parallel real-time program with a hard deadline on the minimum completion time.

From previous results, we know that for systems containing one cluster, the completion time using any schedule where all processors share the same ready queue cannot be more than twice the minimal completion time [4]. For systems with multiple clusters, previous work shows that, in some restricted cases, optimal schedules can be found in polynomial time, e.g., when the number of clusters is limited to two [15], or when there are strong restrictions on the way processes may be scheduled [2]. Some tight performance bounds are known when all processes are independent, i.e., when there are no synchronizations [3], [5], [7], [8].

Two optimal bounds on the minimum completion time and the maximum speedup for multiprocessors with multiple clusters have been reported recently. One of these bounds requires complete knowledge of the parallel profile vector corresponding to the program [12]. This kind of input 
is, in most cases, not available. The other method only uses information from one previous execution [13], thus limiting the information about the parallel program and making the bounds less tight than the ones reported here.

\section{Conclusions}

The two main results in this paper are the functions $t(n, q, \bar{\theta}, \bar{\sigma}, \bar{y}, k, u, \psi)$ and $s(q, \bar{\theta}, \bar{\sigma}, \bar{y}, k, u, \psi)$. These results put nontrivial upper bounds on the minimal completion time of a parallel program, thus making it possible to evaluate heuristic schedules; i.e., if the completion time using a certain heuristic schedule is above the bound, we know that it is worthwhile to look for other schedules. The bounds are based on information from previous executions using different multiprocessors with one cluster.

If there is only information from one previous execution $(q=1)$, the bounds are optimal and, thus, two final results that cannot be improved within the definition of the problem. Consequently, based on the information we have, the bounds cannot be tighter than $t(n, q, \bar{\theta}, \bar{\sigma}, \bar{y}, k, u, \psi)$ and $s(q, \bar{\theta}, \bar{\sigma}, \bar{y}, k, u, \psi)$ when $q=1$. As $q$ increases, the set of programs corresponding to $n, q, \bar{\theta}, \bar{\sigma}$, and $\bar{y}$ becomes smaller, hence, the bounds become tighter in the sense that

$$
t(n, q, \bar{\theta}, \bar{\sigma}, \bar{y}, k, u, \psi) \leq t\left(n, 1, \theta_{1}, \sigma_{1}, y_{1}, k, u, \psi\right)
$$

and

$$
s(q, \bar{\theta}, \bar{\sigma}, \bar{y}, k, u, \psi) \leq s\left(1, \theta_{1}, \sigma_{1}, y_{1}, k, u, \psi\right)
$$

when $q>1$. However, we cannot guarantee that

$$
t(n, q, \bar{\theta}, \bar{\sigma}, \bar{y}, k, u, \psi)
$$

and

$$
s(q, \bar{\theta}, \bar{\sigma}, \bar{y}, k, u, \psi)
$$

are optimal when $q>1$, i.e., based on the information we have it may be possible to obtain bounds which are somewhat tighter than $t(n, q, \bar{\theta}, \bar{\sigma}, \bar{y}, k, u, \psi)$ and $s(q, \bar{\theta}, \bar{\sigma}, \bar{y}, k, u, \psi)$ when $q>1$.

The bounds are based on the assumption that the length and the order of the sequential segments in the parallel program are independent of the way the program is scheduled. This assumption is valid for a number of programs, ranging from parallel real time systems to traditional computer intensive applications such as parallel programs for sorting, matrix manipulation, and the prime sieve program discussed in Section 3. The results are also based on the assumption of zero synchronization overhead. If the program parallelism is relatively coarse grained, this is a reasonable assumption, because the synchronization overhead will be very small compared to the length of the segments of sequential processing. The assumption of zero overhead is also used in most previously reported performance bounds [2], [3], [4], [5], [7], [8], [15].

The results can also be used for selecting a multiprocessor configuration with a guaranteed performance, e.g., when selecting a configuration for a parallel real-time program with a hard deadline on the minimum completion time.

\section{REFERENCES}

[1] M.S. Bazaraa and J.J. Jarvis, Linear Programming and Network Flows. Wiley, 1977.

[2] S.H. Bokhari, "Partitioning Problems in Parallel, Pipelined, and Distributed Computing," IEEE Trans. Computers, vol. 37, no. 1, pp. 48-57, Jan. 1988.

[3] E.G. Coffman Jr., M.R. Garey, and D.S. Johnson, "An Application of Bin Packing to Multiprocessor Scheduling," SIAM J. Computing, vol. 7, no. 1, pp. 1-17, Feb. 1978.

[4] R.L. Graham, "Bounds on Multiprocessor Timing Anomalies," SIAM J. Applied Mathematics, vol. 17, no. 2, pp. 416-429, 1969.

[5] D.K. Friesen, "Tighter Bounds for the Multifit Processor Scheduling Algorithm," SIAM J. Computing, vol. 13, pp. 170-181, 1984.

[6] M. Garey and D. Johnson, Computers and Intractability. W.H. Freeman and Company, 1979.

[7] D.S. Hochbaum and D.B. Shmoys, "Using Dual Approximation Algorithms for Scheduling Problems: Theoretical and Practical Results," J. ACM, vol. 34, no. 1, pp. 144-162, 1987.

[8] M.A. Langston, "Processor Scheduling with Improved Heuristic Algorithms," PhD thesis, Univ. of Texas, College Station, 1981.

[9] H. Lennerstad and L. Lundberg, "An Optimal Execution Time Estimate of Static versus Dynamic Allocation in Multiprocessor Systems," SIAM J. Computing, vol. 24, no. 4, pp. 751-764, Aug. 1995.

[10] L. Lundberg, "Multiprocessor Performance Evaluation of Billing Gateway Systems for Telecommunication Applications," Proc. ISCA Int'l Conf. Parallel and Distributed Computing Systems, pp. 225231, Dijon, France, Sept., 1996.

[11] L. Lundberg, "Predicting the Speedup of Parallel Ada Programs," Proc. Ada Europe Int'l Conf., pp. 257-274, Amsterdam. SpringerVerlag, 1992.

[12] L. Lundberg and H. Lennerstad, "An Optimal Upper Bound on the Minimal Completion Time in Distributed Supercomputing," Proc. 1994 ACM Int'l Conf. Supercomputing, pp. 196-203, Manchester, England, July 1994.

[13] L. Lundberg and H. Lennerstad, "An Optimal Lower Bound on the Maximum Speedup in Multiprocessors with Clusters," Proc. IEEE First Int'l Conf. Algorithms and Architectures for Parallel Processing, pp. 640-649, Brisbane, Australia, Apr. 1995.

[14] L. Lundberg and M. Roos, "Predicting the Speedup of Multithreaded Solaris Programs," Proc. IEEE Conf. High Performance Computing, pp. 386-392, Bangalore, India, Dec. 1997.

[15] H.S. Stone, "Multiprocessor Scheduling with the Aid of Network Flow Algorithms," IEEE Trans. Software Eng., vol. 3, no. 1, pp. 8593, 1977.

[16] SunSoft, Solaris Multithreaded Programming Guide. Prentice Hall, 1995.

[17] http://www.sun.com/microelectronics/datasheets/stp1020a /01.html.

[18] http://www.sun.com/software/Developer-products/ sig/threads.

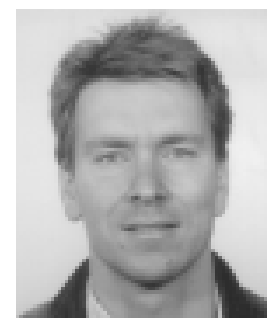

Lars Lundberg received his MS degree in computer science from Linköping University, Sweden, in 1986, and his PhD degree in computer engineering from Lund University, Sweden, in 1993 . He is currently an associate professor of computer systems engineering at the University of Karlskrona/Ronneby, Sweden. He is also head of the computer science department at the University of Karlskrona/Ronneby. Dr. Lundberg's current research interests are in the areas of parallel program scheduling, performance bounds, and software architectures for high performance parallel systems.

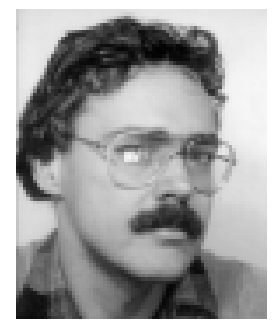

Håkan Lennerstad defended his $\mathrm{PhD}$ thesis in mathematics in 1990 at Chalmers University of Technology, Sweden. He has his MS in electrical engineering. The PhD thesis deals with partial differential equations: the solvability of the Laplace equation in the case of domains with low regularity. $\mathrm{He}$ is now an assistant professor in mathematics at University of Karlskrona/Ronneby, Sweden. There, his main research projects have been on combinatorics for computer system organization. He has also written papers on coding theory and signal processing in collaboration with applied researchers. 